\title{
The Long-Term Effect of Exposure to Particulate Matter Air Pollution on the Incidence of Myocardial Infarction: A Systematic Review and Meta-Analysis
}

\section{Masoud Khosravipour}

Kermanshah University of Medical Sciences

\section{Roya Safari-Faramani}

Kermanshah University of Medical Sciences

Fatemeh Rajati ( $\nabla$ f.rajati@kums.ac.ir)

Kermanshah University of Medical Sciences

Fariborz Omidi

Kermanshah University of Medical Sciences

\section{Research Article}

Keywords: Meta-Analysis, Particulate Matter, Air Pollution, Myocardial Infarction, Heart Attack, Acute Coronary Syndrome Posted Date: April 26th, 2021

DOI: https://doi.org/10.21203/rs.3.rs-440374/v1

License: (c) This work is licensed under a Creative Commons Attribution 4.0 International License. Read Full License 


\section{Abstract}

This study systematically reviews the long-term impact of exposure to particulate matter (PM) air pollution with aerodynamic diameter $\leq 10 \mu \mathrm{m}$ on the incidence of myocardial infarction (MI).

The relevant databases were searched with appropriate keywords on February 29, 2020. A random-effects model through a generic inverse-variance method was used to calculate the pooled hazard ratio (HR) and 95\% confidence interval (Cl) of MI. The number of 17 cohort studies with more than 18 million participants and 800,000 cases of MI were included. A significantly higher risk of MI was observed per $1 \mu \mathrm{g} / \mathrm{m}^{3}$ increment of PM with aerodynamic diameter $\leq 10 \mu \mathrm{m}(\mathrm{HR}=$ $1.02,95 \% \mathrm{Cl}=1.01,1.03)$. Subgroup analysis according to the study population indicates subjects with cardiovascular diseases history had a significantly greater risk of MI per $1 \mu \mathrm{g} / \mathrm{m}^{3}$ increase in PM with aerodynamic diameter $\leq 10 \mu \mathrm{m}$ level $(\mathrm{HR}=1.05,95 \% \mathrm{Cl}=1.01,1.08)$. Subgroup analysis according to aerodynamic diameter of $\mathrm{PM}$ showed only a significant stronger risk of $\mathrm{Ml}$ per $1 \mu \mathrm{g} / \mathrm{m}^{3}$ increase in $\mathrm{PM}$ with aerodynamic diameter $<2.5 \mu \mathrm{m}(\mathrm{HR}=1.01,95 \% \mathrm{Cl}=1.00$, 1.02).

The pooled result confirms a significant association between the long-term exposure to PM air pollution and the developing of MI.

\section{Introduction}

Air pollution is a growing challenge in the world that seriously threatens health of people. According to the World Health Organization (WHO), more than $90 \%$ of the world's population, especially in low-income and middle-income countries lives in areas with inappropriate air pollution quality ${ }^{1}$. Although Air pollution is a complex cocktail of chemicals, more attentions are focused on particulate matter (PM) component mainly due to its high toxicity for lung and cardiovascular system ${ }^{2}$. According to recent data, from 1990 to 2017, global age-standardized summary exposure value for ambient PM air pollution raised by $41.2 \%$ averagely making it the first environmental risk factor in $2017^{3}$.

A large number of adverse health effects can be associated with PM air pollution. Previous epidemiological studies indicted exposure to PM air pollution increased risk of lung disturbances involving mainly chronic obstructive pulmonary disease (COPD) ${ }^{4}$, asthma ${ }^{5}$, and lung cancer ${ }^{6}$ and cardiovascular diseases, including heart failure ${ }^{7}$, hypertension ${ }^{8}$, arthrosclerosis ${ }^{9}$, arrhythmia ${ }^{10}$, carotid intima-media thickness ${ }^{11}$, and stroke ${ }^{12-13}$. Furthermore, metabolic dysfunctions such as diabetes ${ }^{14-15}$, neurological disorders ${ }^{16}$, including Parkinson's disease, dementia, Alzheimer's disease depression and Autism spectrum disorder (ASD) were reported. What is more, some studies indicated PM can be associated with the stronger risk of depression ${ }^{17}$ and kidney disease ${ }^{18}$. The Other studies showed a significant association between PM air pollution and incidence of cancers ${ }^{6,19}$. What is more, both short and long-term exposure to PM air pollution were showed increased risk of mortality and largely respiratory and cardiovascular mortality $20-21$. From 1990 to 2017, the number of deaths attributed to PM air pollution was increased nearly by $68 \%$ and reached to about 5 $\%$ of all deaths ${ }^{3}$.

To current date, many studies have assessed the short-term effect of exposure to PM on MI. According to recent metaanalysis studies, short-term exposure to PM can be significantly associated with the stronger risk of $\mathrm{MI}^{22-25}$. However, the long-term effect of exposure to PM on $\mathrm{MI}$ is inconclusive or even contradictory. Although some studies showed the greater risk of $\mathrm{MI}$ per increase in PM levels ${ }^{26-33}$, others did not find a significant association ${ }^{34-42}$. We designed this systematic and meta-analysis with the aim of synthesizing the current evidence to investigate the long-term association between PM air pollution and the incidence of MI. 


\section{Methods}

\subsection{Protocol and registration}

We acted upon the Preferred Reporting Items for Systematic Reviews and Meta-Analyses statement ${ }^{43}$. Also, the elaboration and explanation of PRISMA statement were considered ${ }^{44}$. This study was approved by Research Deputy and Technology of Kermanshah University of Medical Sciences.

\subsection{Eligibility criteria}

Study population was defined any subjects whose exposure to PM determined and then followed by time for MI occurrence. The hazard ratio (HR) of both primary and secondary MI defined by ICD-9 code 410 or ICD-10 codes I21 to I23 was considered outcome. It should be noted we excluded studies with including ICD-10 codes I20, I24, and I25 under name of ischemic heart disease (IHD) or coronary heart disease (CHD) if did not provide an independent HR for MI. Given that the focus of this study was in clarifying the long-term effect of PM on Ml, we included only prospective cohort studies. No publication date restriction was considered, however, we entered studies with English language.

\subsection{Information sources}

On July 14, 2019, we searched PubMed (https://www.ncbi.nlm.nih.gov/pubmed/), Scopus (https://www.scopus.com/), and Web of Science (https://www.webofknowledge.com/) to trace any relevant publication. The last updating for new articles was performed on February 29, 2020. We also screened references of included studies to find any relevant publication that may not be detected by the searching.

\subsection{Search}

We searched the mentioned databases by appropriate keywords and terms such as "myocardial infarction" ,"heart attack", "acute coronary syndrome" as outcome, and "air pollution", "air pollutants", "particulate matter", and "PM" as exposure. Then, Endnote files of records were obtained for further assessment.

\subsection{Study selection}

As illustrated in Fig. 1, after removing duplicate records, we screened documents by title and abstract then excluded studies did not investigated the association. Next, we retrieved the full text of potentially relevant studies and examined them carefully. According to the eligibility criteria, at first, studies did not investigated PM as an exposure or MI as an outcome were omitted. Second, studies assessed the short-term effect of air pollution on MI were removed. Third, we discarded studies assessed the effects of PM on MI mortality or studies mixed the incidence and death of MI. Forth, we removed studies with including ICD-10 codes I20, I24, and I25 under name of ischemic heart disease (IHD) or coronary heart disease (CHD) because did not provide an independent HR for MI. Finally, book chapters, conference papers, editorials, and review articles were eliminated.

\subsection{Data collection process}

We assessed carefully all included studies. The main characteristics of studies recorded in Microsoft excel version 2013. During the process of data collection whenever there was a problem, we solved it with team-based discussion or consulted with other available researchers or the relevant books.

\subsection{Data items}

The following items were extracted from each study: study ID (name and publication year), country that study was conducted, characteristics of study population (age, sex, and sample size), PM measurement methods and types of 
them, the way of diagnosing $\mathrm{Ml}$, and the estimated $\mathrm{HR}$ and 95\% $\mathrm{Cl}$ (confidence interval)of $\mathrm{MI}$ with adjusted variables (see Table 1). 
Table 1

The summary characteristics of included studies.

\begin{tabular}{|c|c|c|c|c|c|c|}
\hline Study ID & Country & $\begin{array}{l}\text { Study } \\
\text { characteristics }\end{array}$ & $\begin{array}{l}\text { Particulate } \\
\text { measurement }\end{array}$ & $\begin{array}{l}\text { MI } \\
\text { assessment }\end{array}$ & Risk measurement & $\begin{array}{l}\text { Risk } \\
\text { of } \\
\text { bias }\end{array}$ \\
\hline $\begin{array}{l}\text { Miller et } \\
\text { al. } \\
(2007)\end{array}$ & $\begin{array}{l}\text { The United } \\
\text { Stated of } \\
\text { America } \\
\text { (USA) }\end{array}$ & $\begin{array}{l}\text { Target } \\
\text { Population: } \\
\text { postmenopausal } \\
\text { women } \\
\text { Sample size: } \\
58,610 \\
\text { Follow } \\
\text { up:1994-2002 } \\
\text { Sex: F } \\
\text { Age: } 50-79 \\
\text { years }\end{array}$ & $\begin{array}{l}\text { Source of data } \\
\text { :The } \\
\text { Environmental } \\
\text { Protection } \\
\text { Agency's } \\
\text { Aerometric } \\
\text { Information } \\
\text { Retrieval } \\
\text { System } \\
\text { Exposure } \\
\text { assessment: } \\
\text { The nearest } \\
\text { monitor } \\
\text { according to } \\
\text { ZIP Code } \\
\text { Type: Annual } \\
\text { averages of } \\
\text { PM }{ }_{2.5}\end{array}$ & $\begin{array}{l}\text { Number of } \\
\text { cases: } 584 \\
\text { Diagnose: } \\
\text { Questionnaires } \\
\text { and the review } \\
\text { of medical } \\
\text { records by } \\
\text { physician } \\
\text { adjudicators }\end{array}$ & $\begin{array}{l}\text { Index: } \mathrm{HR}(95 \% \mathrm{Cl}) \text { per } \\
10 \mu \mathrm{g} / \mathrm{m}^{3} \text { increment } \\
\text { of PM } \\
\text { Adjustment: age, } \\
\text { race or ethnic group, } \\
\text { educational level, } \\
\text { household income, } \\
\text { smoking status, } \\
\text { systolic blood } \\
\text { pressure, body-mass } \\
\text { index, and presence } \\
\text { or absence of } \\
\text { diabetes, } \\
\text { hypertension, or } \\
\text { hypercholesterolemia }\end{array}$ & Good \\
\hline $\begin{array}{l}\text { Zanobetti } \\
\text { et al. } \\
(2007)\end{array}$ & USA & $\begin{array}{l}\text { Target } \\
\text { Population: } \\
\text { Patients with } \\
\text { myocardial } \\
\text { infarction (MI) } \\
\text { Sample size: } \\
\text { 196,131 } \\
\text { Follow } \\
\text { up:1985-1999 } \\
\text { Sex: M\&F } \\
\text { Age: } \geq 65 \text { years }\end{array}$ & $\begin{array}{l}\text { Source of } \\
\text { data: The } \\
\text { Environmental } \\
\text { Protection } \\
\text { Agency's } \\
\text { Aerometric } \\
\text { Information } \\
\text { Retrieval } \\
\text { System } \\
\text { Exposure } \\
\text { assessment: } \\
\text { cities levels }\end{array}$ & $\begin{array}{l}\text { Number of } \\
\text { cases: } 22,522 \\
\text { Diagnose: } \\
\text { Medical } \\
\text { records based } \\
\text { on ICD-9: } 410\end{array}$ & $\begin{array}{l}\text { Index: } \mathrm{HR}(95 \% \mathrm{Cl}) \text { per } \\
10 \mu \mathrm{m} / \mathrm{m}^{3} \text { increment } \\
\text { of PM } \\
\text { Adjustment: time } \\
\text { period, age, sex, race, } \\
\text { and type of MI. } \\
\text { model controlled for } \\
\text { days of coronary } \\
\text { care and intensive } \\
\text { care, previous } \\
\text { diagnosis for atrial } \\
\text { fibrillation, and } \\
\text { secondary or } \\
\text { previous diagnoses } \\
\text { for COPD, diabetes, } \\
\text { and hypertension }\end{array}$ & Good \\
\hline
\end{tabular}




\begin{tabular}{|c|c|c|c|c|c|c|}
\hline Study ID & Country & $\begin{array}{l}\text { Study } \\
\text { characteristics }\end{array}$ & $\begin{array}{l}\text { Particulate } \\
\text { measurement }\end{array}$ & $\begin{array}{l}\text { MI } \\
\text { assessment }\end{array}$ & Risk measurement & $\begin{array}{l}\text { Risk } \\
\text { of } \\
\text { bias }\end{array}$ \\
\hline $\begin{array}{l}\text { Puett et al. } \\
(2008)\end{array}$ & USA & $\begin{array}{l}\text { Target } \\
\text { Population: } \\
\text { Nurses } \\
\text { Sample size: } \\
66,250 \\
\text { Follow } \\
\text { up:1992-2002 } \\
\text { Sex: F } \\
\text { Age: } 30-55 \\
\text { years }\end{array}$ & $\begin{array}{l}\text { Source of } \\
\text { data: The } \\
\text { Environmental } \\
\text { Protection } \\
\text { Agency's } \\
\text { Aerometric } \\
\text { Information } \\
\text { Retrieval } \\
\text { System, the } \\
\text { IMPROVE } \\
\text { network, and } \\
\text { Harvard } \\
\text { University } \\
\text { research } \\
\text { studies. } \\
\text { Exposure } \\
\text { assessment: A } \\
\text { geographic } \\
\text { information } \\
\text { system-based } \\
\text { spatial } \\
\text { smoothing } \\
\text { model } \\
\text { Type: Monthly } \\
\text { averages of } \\
\text { PM } 10\end{array}$ & $\begin{array}{l}\text { Number of } \\
\text { cases: } 854 \\
\text { Diagnose: } \\
\text { World Health } \\
\text { Organization } \\
\text { criteria } \\
\text { through } \\
\text { interview or } \\
\text { confirmed } \\
\text { cases by } \\
\text { hospitals }\end{array}$ & $\begin{array}{l}\text { Index: } \mathrm{HR}(95 \% \mathrm{Cl}) \text { per } \\
10 \mu \mathrm{g} / \mathrm{m}^{3} \text { increment } \\
\text { of PM } \\
\text { Adjustment: } \\
\text { Stratifying by age in } \\
\text { months, adjusting for } \\
\text { state of residence, } \\
\text { year, and season. }\end{array}$ & Good \\
\hline $\begin{array}{l}\text { Puett et al. } \\
\text { (2009) }\end{array}$ & UAS & $\begin{array}{l}\text { Target } \\
\text { Population: } \\
\text { Nurses } \\
\text { Sample size: } \\
66,250 \\
\text { Follow } \\
\text { up:1992-2002 } \\
\text { Sex: F } \\
\text { Age: } 30-55 \\
\text { years }\end{array}$ & $\begin{array}{l}\text { Source of } \\
\text { data: The } \\
\text { Environmental } \\
\text { Protection } \\
\text { Agency's } \\
\text { Aerometric } \\
\text { Information } \\
\text { Retrieval } \\
\text { System, the } \\
\text { IMPROVE } \\
\text { network, and } \\
\text { Harvard } \\
\text { University } \\
\text { research } \\
\text { studies. } \\
\text { Exposure } \\
\text { assessment: A } \\
\text { geographic } \\
\text { information } \\
\text { system-based } \\
\text { spatial } \\
\text { smoothing } \\
\text { model } \\
\text { Type: Monthly } \\
\text { averages of } \\
\text { PM } 2.5 \text { and PM } \\
2.5-10\end{array}$ & $\begin{array}{l}\text { Number of } \\
\text { cases: } 854 \\
\text { Diagnose: } \\
\text { World Health } \\
\text { Organization } \\
\text { criteria } \\
\text { through } \\
\text { interview or } \\
\text { confirmed } \\
\text { cases by } \\
\text { hospitals }\end{array}$ & $\begin{array}{l}\text { Index: } \mathrm{HR}(95 \% \mathrm{Cl}) \text { per } \\
10 \mu \mathrm{g} / \mathrm{m}^{3} \text { increment } \\
\text { of PM } \\
\text { Adjustment: } \\
\text { Stratifying by age in } \\
\text { months, adjusting for } \\
\text { state of residence, } \\
\text { year, and season. }\end{array}$ & Good \\
\hline
\end{tabular}




\begin{tabular}{|c|c|c|c|c|c|c|}
\hline Study ID & Country & $\begin{array}{l}\text { Study } \\
\text { characteristics }\end{array}$ & $\begin{array}{l}\text { Particulate } \\
\text { measurement }\end{array}$ & $\begin{array}{l}\text { MI } \\
\text { assessment }\end{array}$ & Risk measurement & $\begin{array}{l}\text { Risk } \\
\text { of } \\
\text { bias }\end{array}$ \\
\hline $\begin{array}{l}\text { Lipsett et } \\
\text { al.(2011) }\end{array}$ & USA & $\begin{array}{l}\text { Target } \\
\text { Population: } \\
\text { Current and } \\
\text { former public } \\
\text { school } \\
\text { professionals } \\
\text { (Teachers) } \\
\text { Sample size: } \\
\text { 124,614 } \\
\text { Follow } \\
\text { up:1997-2005 } \\
\text { Sex: F } \\
\text { Age: } \geq 20 \text { years }\end{array}$ & $\begin{array}{l}\text { Source of } \\
\text { data: Federal } \\
\text { Reference } \\
\text { Method } \\
\text { monitors of } \\
\text { California's } \\
\text { State, the } \\
\text { Local Air } \\
\text { Monitoring } \\
\text { Network and } \\
\text { the } \\
\text { Interagency } \\
\text { Monitoring of } \\
\text { Protected } \\
\text { Visual } \\
\text { Environments } \\
\text { (IMPROVE) } \\
\text { network } \\
\text { Exposure } \\
\text { assessment: } \\
\text { Geocoded } \\
\text { residential } \\
\text { addresses } \\
\text { with inverse } \\
\text { distance- } \\
\text { weighted } \\
\text { Type: Monthly } \\
\text { averages of } \\
\text { PM } 2.5 \text { and } \\
\text { PM } 10\end{array}$ & $\begin{array}{l}\text { Number of } \\
\text { cases: } 722 \\
\text { Diagnose: } \\
\text { Government } \\
\text { mortality and } \\
\text { hospitalization } \\
\text { Files } \\
\text { according to } \\
\text { ICD-10: I21 }\end{array}$ & $\begin{array}{l}\text { Index: HR(95\%Cl) per } \\
10 \mu \mathrm{g} / \mathrm{m}^{3} \text { increment } \\
\text { of PM } \\
\text { Adjustment: age, } \\
\text { race, smoking status, } \\
\text { total pack-years, } \\
\text { body mass index, } \\
\text { marital status, } \\
\text { alcohol } \\
\text { consumption, } \\
\text { second-hand smoke } \\
\text { exposure at home, } \\
\text { dietary fat, dietary } \\
\text { fiber, dietary calories, } \\
\text { physical activity, } \\
\text { menopausal status, } \\
\text { hormone therapy } \\
\text { use, family history of } \\
\text { Ml or stroke, blood } \\
\text { pressure medication, } \\
\text { and aspirin use, and } \\
\text { for contextual } \\
\text { variables (income, } \\
\text { income inequality, } \\
\text { education, } \\
\text { population size, } \\
\text { racial composition, } \\
\text { and unemployment) }\end{array}$ & Good \\
\hline $\begin{array}{l}\text { Atkinson } \\
\text { et al. } \\
(2013)\end{array}$ & $\begin{array}{l}\text { The United } \\
\text { Kingdom } \\
\text { (UK) }\end{array}$ & $\begin{array}{l}\text { Target } \\
\text { Population: } \\
\text { General } \\
\text { Sample size: } \\
836,557 \\
\text { Follow up:2003- } \\
07 \\
\text { Sex: M\&F } \\
\text { Age: } 40 \text { to } 89 \\
\text { years }\end{array}$ & $\begin{array}{l}\text { Source of } \\
\text { data: The } \\
\text { national } \\
\text { emission } \\
\text { inventories } \\
\text { (NAEI) } \\
\text { Exposure } \\
\text { assessment: } \\
\text { air dispersion } \\
\text { models } \\
\text { Type: Annual } \\
\text { averages of } \\
\text { PM }_{10}\end{array}$ & $\begin{array}{l}\text { Number of } \\
\text { cases: } 13,956 \\
\text { Diagnose: } \\
\text { Reviewing } \\
\text { medical } \\
\text { records based } \\
\text { on ICD-10: I21- } \\
23\end{array}$ & $\begin{array}{l}\text { Index: HR(95\%Cl) per } \\
3 \mu \mathrm{g} / \mathrm{m}^{3} \text { increment } \\
\text { of PM } \\
\text { Adjustment: age and } \\
\text { sex, smoking, BMI, } \\
\text { Diabetes and } \\
\text { hypertension, IMD }\end{array}$ & Good \\
\hline
\end{tabular}




\begin{tabular}{|c|c|c|c|c|c|c|}
\hline Study ID & Country & $\begin{array}{l}\text { Study } \\
\text { characteristics }\end{array}$ & $\begin{array}{l}\text { Particulate } \\
\text { measurement }\end{array}$ & $\begin{array}{l}\text { MI } \\
\text { assessment }\end{array}$ & Risk measurement & $\begin{array}{l}\text { Risk } \\
\text { of } \\
\text { bias }\end{array}$ \\
\hline $\begin{array}{l}\text { Koton et } \\
\text { al. } \\
(2013)\end{array}$ & Israel & $\begin{array}{l}\text { Target } \\
\text { Population: } \\
\text { Patients with MI } \\
\text { Sample size: } \\
1120 \\
\text { Follow } \\
\text { up:1992-2005 } \\
\text { Sex: M\&F } \\
\text { Age: } \leq 65 \text { years }\end{array}$ & $\begin{array}{l}\text { Source of } \\
\text { data: Air- } \\
\text { quality } \\
\text { monitoring } \\
\text { stations in } \\
\text { central Israel } \\
\text { Exposure } \\
\text { assessment: } \\
\text { Geocoded } \\
\text { residential } \\
\text { addresses and } \\
\text { linear } \\
\text { regression } \\
\text { model to } \\
\text { estimate } \\
\text { PM }_{2.5} \text { from } \\
\text { PM }_{10} \\
\text { Type: Annual }^{\text {averages of }} \\
\text { PM }_{2.5}\end{array}$ & $\begin{array}{l}\text { Number of } \\
\text { cases: } 341 \\
\text { Diagnose: } \\
\text { Medical } \\
\text { records by a } \\
\text { senior } \\
\text { cardiologist }\end{array}$ & $\begin{array}{l}\text { Index: HR( } 95 \% \mathrm{Cl}) \text { per } \\
10 \mu \mathrm{\mu g} / \mathrm{m}^{3} \text { increment } \\
\text { of PM } \\
\text { Adjustment: age and } \\
\text { gender, smoking, } \\
\text { diabetes, } \\
\text { hypertension, } \\
\text { dyslipidemia, leisure } \\
\text { time physical activity, } \\
\text { Killip class, } \\
\text { thrombolysis, } \\
\text { coronary artery } \\
\text { bypass graft surgery } \\
\text { within } 45 \text { days, } \\
\text { percutaneous } \\
\text { coronary angioplasty } \\
\text { within } 45 \text { days, } \\
\text { Charlson index and } \\
\text { chronic CHD, } \\
\text { education, income, } \\
\text { employment, } \\
\text { neighborhood SES. } \\
\text { Employment. }\end{array}$ & Good \\
\hline $\begin{array}{l}\text { Nishiwaki } \\
\text { et al. } \\
(2013)\end{array}$ & Japan & $\begin{array}{l}\text { Target } \\
\text { Population: } \\
\text { middle-aged } \\
\text { Japanese } \\
\text { Sample size: } \\
62,142 \\
\text { Follow } \\
\text { up:1990-2005 } \\
\text { Sex: M\&F } \\
\text { Age: 40-69 } \\
\text { years }\end{array}$ & $\begin{array}{l}\text { Source of } \\
\text { data: The } \\
\text { Ministry of the } \\
\text { Environment } \\
\text { Exposure } \\
\text { assessment: } \\
\text { The nearest } \\
\text { station to } \\
\text { postal address } \\
\text { Type: Annual } \\
\text { averages of } \\
\text { PM }_{10}\end{array}$ & $\begin{array}{l}\text { Number of } \\
\text { cases: NC. } \\
\text { Diagnose: } \\
\text { Questionnaire } \\
\text { and reviewing } \\
\text { medical } \\
\text { records } \\
\text { according to } \\
\text { MTDCT } \\
\text { criteria. }\end{array}$ & $\begin{array}{l}\text { Index: HR(95\%Cl) per } \\
10 \mu g / \mathrm{m}^{3} \text { increment } \\
\text { of PM } \\
\text { Adjustment: age, sex, } \\
\text { body mass index, } \\
\text { smoking status, } \\
\text { environmental } \\
\text { smoking status, and } \\
\text { alcohol } \\
\text { consumption. }\end{array}$ & Good \\
\hline $\begin{array}{l}\text { Cary et al. } \\
(2019)\end{array}$ & UK & $\begin{array}{l}\text { Target } \\
\text { Population: } \\
\text { General } \\
\text { Sample size: } \\
207042 \\
\text { Follow up: } 2005- \\
11 \\
\text { Sex: M\&F } \\
\text { Age: } 40-79 \\
\text { years }\end{array}$ & $\begin{array}{l}\text { Source of } \\
\text { data: London } \\
\text { Atmospheric } \\
\text { Emissions } \\
\text { Inventory } \\
\text { Exposure } \\
\text { assessment: } \\
\text { KCLurban } \\
\text { dispersion } \\
\text { model } \\
\text { Type: Annual } \\
\text { averages of } \\
\text { PM }_{2.5}\end{array}$ & $\begin{array}{l}\text { Number of } \\
\text { cases: } 2582 \\
\\
\text { Diagnose: } \\
\text { Medical } \\
\text { records based } \\
\text { on ICD-10: I21- } \\
23\end{array}$ & $\begin{array}{l}\text { Index: HR( } 95 \% \mathrm{Cl}) \text { per } \\
1 \mu \mathrm{m} / \mathrm{m}^{3} \text { increment } \\
\text { of PM } \\
\text { Adjustment: age, } \\
\text { gender, smoking and } \\
\text { BMI, IMD. }\end{array}$ & Good \\
\hline
\end{tabular}




\begin{tabular}{|c|c|c|c|c|c|c|}
\hline Study ID & Country & $\begin{array}{l}\text { Study } \\
\text { characteristics }\end{array}$ & $\begin{array}{l}\text { Particulate } \\
\text { measurement }\end{array}$ & $\begin{array}{l}\text { MI } \\
\text { assessment }\end{array}$ & Risk measurement & $\begin{array}{l}\text { Risk } \\
\text { of } \\
\text { bias }\end{array}$ \\
\hline $\begin{array}{l}\text { Hartiala et } \\
\text { al. } \\
(2016)\end{array}$ & USA & $\begin{array}{l}\text { Target } \\
\text { Population: } \\
\text { patients } \\
\text { undergoing } \\
\text { elective } \\
\text { diagnostic } \\
\text { coronary } \\
\text { angiography. } \\
\text { Sample size: } \\
5854 \\
\text { Follow up: } 3 \\
\text { years } \\
\text { Sex: M\&F } \\
\text { Mean Age (SD): } \\
64 \text { (11) }\end{array}$ & $\begin{array}{l}\text { Source of } \\
\text { data: The US } \\
\text { Environmental } \\
\text { Protection } \\
\text { Agency's (EPA) } \\
\text { Air Quality } \\
\text { System (AQS) } \\
\text { database } \\
\text { Exposure } \\
\text { assessment: } \\
\text { Zip code by } \\
\text { inverse } \\
\text { distance- } \\
\text { squared } \\
\text { weighting. } \\
\text { Type: Monthly } \\
\text { average of } \\
\text { PM }{ }_{2.5}\end{array}$ & $\begin{array}{l}\text { Number of } \\
\text { cases: } 288 \\
\\
\text { Diagnose: } \\
\text { Verified } \\
\text { medical } \\
\text { records }\end{array}$ & $\begin{array}{l}\text { Index: } \mathrm{HR}(95 \% \mathrm{Cl}) \text { per } \\
1 \mu \mathrm{m} / \mathrm{m}^{3} \text { increment } \\
\text { of PM } \\
\text { Adjustment: age, sex, } \\
\text { education level, and } \\
\text { current smoking. }\end{array}$ & Good \\
\hline $\begin{array}{l}\text { Kim et al. } \\
\text { (2017) }\end{array}$ & Korea & $\begin{array}{l}\text { Target } \\
\text { Population: } \\
\text { General } \\
\text { Sample size: } \\
136094 \\
\text { Follow up:2007- } \\
13 \\
\text { Sex: M\&F } \\
\text { Age: } \geq 18 \text { years }\end{array}$ & $\begin{array}{l}\text { Source of } \\
\text { data: The } \\
\text { Korean } \\
\text { Ministry of } \\
\text { Environment } \\
\text { Exposure } \\
\text { assessment: } \\
\text { ZIP code } \\
\text { Type: Monthly } \\
\text { average of } \\
\mathrm{PM}_{2.5} \text { and } \\
\mathrm{PM}_{10}\end{array}$ & $\begin{array}{l}\text { Number of } \\
\text { cases: } 354 \\
\text { Diagnose: ICD- } \\
\text { 10: } 12-123\end{array}$ & $\begin{array}{l}\text { Index: HR(95\%Cl) per } \\
1 \mu \mathrm{\mu g} \mathrm{m}^{3} \text { increment } \\
\text { of PM } \\
\text { Adjustment: age, sex, } \\
\text { socioeconomic } \\
\text { status, hypertension, } \\
\text { diabetes mellitus, } \\
\text { dyslipidemia, chronic } \\
\text { renal failure, end- } \\
\text { stage renal diseases, } \\
\text { ischemic heart } \\
\text { disease, peripheral } \\
\text { arterial disease, } \\
\text { chronic obstructive } \\
\text { pulmonary disease, } \\
\text { and malignancy, } \\
\text { body mass index, } \\
\text { fasting blood } \\
\text { glucose, total } \\
\text { cholesterol, and } \\
\text { hemoglobin. } \\
\text { Composite } \\
\text { cardiovascular } \\
\text { events were a } \\
\text { composite of } \\
\text { cardiovascular death, } \\
\text { acute myocardial } \\
\text { infarction, congestive } \\
\text { heart failure, and } \\
\text { stroke. }\end{array}$ & Good \\
\hline
\end{tabular}




\begin{tabular}{|c|c|c|c|c|c|c|}
\hline Study ID & Country & $\begin{array}{l}\text { Study } \\
\text { characteristics }\end{array}$ & $\begin{array}{l}\text { Particulate } \\
\text { measurement }\end{array}$ & $\begin{array}{l}\text { MI } \\
\text { assessment }\end{array}$ & Risk measurement & $\begin{array}{l}\text { Risk } \\
\text { of } \\
\text { bias }\end{array}$ \\
\hline $\begin{array}{l}\text { Downward } \\
\text { et al. } \\
(2018)\end{array}$ & Netherlands & $\begin{array}{l}\text { Target } \\
\text { Population: } \\
\text { General } \\
\text { Sample size: } \\
33,831 \\
\text { Follow } \\
\text { up:1993-2010 } \\
\text { Sex: M\&F } \\
\text { Age: } 20-70 \\
\text { years }\end{array}$ & $\begin{array}{l}\text { Source of } \\
\text { data: } \\
\text { Measurements } \\
\text { in } 242 \\
\text { monitoring } \\
\text { sites } \\
\text { Exposure } \\
\text { assessment: } \\
\text { LUR models } \\
\text { Types: Annual } \\
\text { averages of } \\
\text { PM }_{2.5}, \text { PM } \\
2.5-10, \text { and } \\
\text { PM }_{10}\end{array}$ & $\begin{array}{l}\text { Number of } \\
\text { cases: } 797 \\
\text { Diagnose: } \\
\text { Medical } \\
\text { records based } \\
\text { on ICD-10: I21- } \\
23\end{array}$ & $\begin{array}{l}\text { Index: HR(95\%Cl) per } \\
5 \mu \mathrm{g} / \mathrm{m}^{3} \text { increment } \\
\text { of } \mathrm{PM}_{2.5} \text { and } \\
\mathrm{PM}_{2.5-10} \text { and per } 10 \\
\mu \mathrm{g} / \mathrm{m} \text { Increment of } \\
\mathrm{PM}_{10} \\
\text { Adjustment: smoking } \\
\text { status (including } \\
\text { number of cigarettes } \\
\text { and duration of } \\
\text { smoking), diet } \\
\text { (intake of fruit and } \\
\text { vegetables), alcohol } \\
\text { consumption, BMI, } \\
\text { recruitment year, } \\
\text { gender, marital } \\
\text { status, education } \\
\text { level, and area-level } \\
\text { economic status. }\end{array}$ & Good \\
\hline $\begin{array}{l}\text { Gandini et } \\
\text { al. } \\
(2018)\end{array}$ & Italy & $\begin{array}{l}\text { Target } \\
\text { Population: } \\
\text { General } \\
\text { Sample size: } \\
74,989 \\
\text { Follow up: } \\
\text { 1999-2008 } \\
\text { Sex: M\&F } \\
\text { Age: > } 35 \text { years }\end{array}$ & $\begin{array}{l}\text { Source of } \\
\text { data: National } \\
\text { Integrated } \\
\text { Modelling } \\
\text { system for } \\
\text { International } \\
\text { Negotiation on } \\
\text { atmospheric } \\
\text { pollution } \\
\text { Exposure } \\
\text { assessment: } \\
\text { atmospheric } \\
\text { chemical } \\
\text { transport } \\
\text { model (CTM) } \\
\text { Type: Annual } \\
\text { average of } \\
\text { PM } 2.5\end{array}$ & $\begin{array}{l}\text { Number of } \\
\text { cases: NC } \\
\text { Diagnose: } \\
\text { Italian } \\
\text { National } \\
\text { Institute of } \\
\text { Statistics } \\
\text { records based } \\
\text { on ICD-9: } 410\end{array}$ & $\begin{array}{l}\text { Index: HR(95\%Cl) per } \\
10 \mu \mathrm{\mu g} / \mathrm{m}^{3} \text { increment } \\
\text { of PM } \\
\text { Adjustment: age, } \\
\text { gender, educational } \\
\text { level, living with } \\
\text { partner, occupational } \\
\text { status, smoking } \\
\text { habit, physical } \\
\text { activity status, type } \\
\text { of municipality (rural, } \\
\text { urban, metropolitan } \\
\text { areas) and BMI. }\end{array}$ & Good \\
\hline
\end{tabular}




\begin{tabular}{|c|c|c|c|c|c|c|}
\hline Study ID & Country & $\begin{array}{l}\text { Study } \\
\text { characteristics }\end{array}$ & $\begin{array}{l}\text { Particulate } \\
\text { measurement }\end{array}$ & $\begin{array}{l}\text { MI } \\
\text { assessment }\end{array}$ & Risk measurement & $\begin{array}{l}\text { Risk } \\
\text { of } \\
\text { bias }\end{array}$ \\
\hline $\begin{array}{l}\text { Loop et al. } \\
\text { (2018) }\end{array}$ & USA & $\begin{array}{l}\text { Target } \\
\text { population: } \\
\text { General } \\
\text { Sample size: } \\
17,126 \\
\text { Follow up: } 2003- \\
12 \\
\text { Sex: M\&F } \\
\text { Age: } \geq 45 \text { years }\end{array}$ & $\begin{array}{l}\text { Source of } \\
\text { data: EPA Air } \\
\text { Quality } \\
\text { System (AQS) } \\
\text { and the } \\
\text { National } \\
\text { Aeronautics } \\
\text { and Space } \\
\text { Administration } \\
\text { (NASA) Aqua } \\
\text { satellite } \\
\text { Exposure } \\
\text { assessment: } \\
\text { Regression } \\
\text { models } \\
\text { Type: Annual } \\
\text { average of } \\
\text { PM }_{2.5}\end{array}$ & $\begin{array}{l}\text { Number of } \\
\text { cases: } 413 \\
\text { Diagnose: } \\
\text { Medical record } \\
\text { review and } \\
\text { interview by } \\
\text { Physicians }\end{array}$ & $\begin{array}{l}\text { Index: } \mathrm{HR}(95 \% \mathrm{Cl}) \mathrm{per} \\
2.7 \mu \mathrm{g} / \mathrm{m}^{3} \text { increment } \\
\text { of PM } \\
\text { Adjustment: 1-year } \\
\text { mean temperature, } \\
\text { season, race, region, } \\
\text { urbanicity, income, } \\
\text { education, age, } \\
\text { gender, pack-years, } \\
\text { BMl, alcohol use, } \\
\text { physical activity, } \\
\text { calendar year, statin } \\
\text { use, antihypertensive } \\
\text { medication use, and } \\
\text { diabetes. }\end{array}$ & Good \\
\hline $\begin{array}{l}\text { Bai et al. } \\
(2019)\end{array}$ & Canada & $\begin{array}{l}\text { Target } \\
\text { Population: } \\
\text { General } \\
\text { Sample size: } \\
5,141,172 \\
\text { Follow up: } 2001- \\
15 \\
\text { Sex: M\&F } \\
\text { Age: } 35 \text { to } 85 \\
\text { years }\end{array}$ & $\begin{array}{l}\text { Source of } \\
\text { data: the } \\
\text { National Air } \\
\text { Pollution } \\
\text { Surveillance } \\
\text { network and } \\
\text { the } \\
\text { Interagency } \\
\text { Monitoring of } \\
\text { Protected } \\
\text { Visual } \\
\text { Environments } \\
\text { network } \\
\text { (IMPROVE) } \\
\text { Exposure } \\
\text { assessment: } \\
\text { global } \\
\text { atmospheric } \\
\text { chemistry } \\
\text { transport } \\
\text { model (GEOS- } \\
\text { Chem CTM), } \\
\text { combined with } \\
\text { a } \\
\text { geographically } \\
\text { weighted } \\
\text { regression } \\
\text { model } \\
\text { Type: Annual } \\
\text { average of } \\
\text { PM } 2.5\end{array}$ & $\begin{array}{l}\text { Number of } \\
\text { cases: } 197,628 \\
\text { Diagnose: } \\
\text { physician- } \\
\text { diagnosed } \\
\text { cases of MI } \\
\text { recorded } \\
\text { obtained from } \\
\text { the Ontario } \\
\text { Myocardial } \\
\text { Infarction } \\
\text { Database (ICD- } \\
\text { 10: I21) }\end{array}$ & $\begin{array}{l}\text { Index: } \mathrm{HR}(95 \% \mathrm{Cl}) \text { per } \\
3.5 \mu \mathrm{m} / \mathrm{m}^{3} \text { increment } \\
\text { of PM } \\
\text { Adjustment: age and } \\
\text { sex only, and } \\
\text { stratified region, } \\
\text { neighborhood -level } \\
\text { recent immigrants } \\
\text { (arrived in the } 5 \text { years } \\
\text { prior to census), } \\
\text { unemployment rate, } \\
\text { education, and } \\
\text { annual household } \\
\text { income, urban } \\
\text { residency and a } \\
\text { north/south indicator }\end{array}$ & Good \\
\hline
\end{tabular}




\begin{tabular}{|c|c|c|c|c|c|c|}
\hline Study ID & Country & $\begin{array}{l}\text { Study } \\
\text { characteristics }\end{array}$ & $\begin{array}{l}\text { Particulate } \\
\text { measurement }\end{array}$ & $\begin{array}{l}\text { MI } \\
\text { assessment }\end{array}$ & Risk measurement & $\begin{array}{l}\text { Risk } \\
\text { of } \\
\text { bias }\end{array}$ \\
\hline \multirow[t]{2}{*}{$\begin{array}{l}\text { Bai et al. } \\
\text { (2019) }\end{array}$} & Canada & $\begin{array}{l}\text { Target } \\
\text { Population : } \\
\text { General } \\
\text { Sample size: } \\
\text { 1,127,209 } \\
\text { Follow up: } \\
\text { 1996-2012 } \\
\text { Sex: M\&F } \\
\text { Age: } 30-100 \\
\text { years }\end{array}$ & $\begin{array}{l}\text { Source of } \\
\text { data: The } \\
\text { National Air } \\
\text { Pollution } \\
\text { Surveillance } \\
\text { network and } \\
\text { the } \\
\text { Interagency } \\
\text { Monitoring of } \\
\text { Protected } \\
\text { Visual } \\
\text { Environments } \\
\text { network } \\
\text { (IMPROVE) } \\
\text { Exposure } \\
\text { assessment: } \\
\text { global } \\
\text { atmospheric } \\
\text { chemistry } \\
\text { transport } \\
\text { model (GEOS- } \\
\text { Chem CTM), } \\
\text { combined with } \\
\text { a } \\
\text { geographically } \\
\text { weighted } \\
\text { regression } \\
\text { model }\end{array}$ & \multirow[t]{2}{*}{$\begin{array}{l}\text { Number of } \\
\text { cases: } 43,745 \\
\text { Diagnose: } \\
\text { physician- } \\
\text { diagnosed } \\
\text { cases of MI } \\
\text { based on } \\
\text { recorded } \\
\text { obtained from } \\
\text { the Ontario } \\
\text { Myocardial } \\
\text { Infarction } \\
\text { Database } \\
\text { according to } \\
\text { ICD-10: I21 }\end{array}$} & \multirow[t]{2}{*}{$\begin{array}{l}\text { Adjustment: age, sex, } \\
\text { tract-level percentage } \\
\text { of recent immigrants, } \\
\text { percentage of the } \\
\text { population aged } \geq \\
15 \text { years without } \\
\text { employment, } \\
\text { percentage of the } \\
\text { population aged } \geq \\
15 \text { years with less } \\
\text { than a high school } \\
\text { education, and } \\
\text { annual household } \\
\text { income, } \\
\text { hypertension, } \\
\text { diabetes, CHF, } \\
\text { chronic obstructive } \\
\text { pulmonary disease, } \\
\text { asthma, and cancer. }\end{array}$} & \multirow[t]{2}{*}{ Gooc } \\
\hline & & & $\begin{array}{l}\text { Type: Annual } \\
\text { average of } \\
\mathrm{PM}_{2.5}\end{array}$ & & & \\
\hline $\begin{array}{l}\text { Danesh } \\
\text { Yazdi et } \\
\text { al. } \\
\text { (2019) }\end{array}$ & USA & $\begin{array}{l}\text { Target } \\
\text { Population: } \\
\text { General } \\
\text { Sample size: } \\
11,084,660 \\
\text { Follow up: } 2000- \\
12 \\
\text { Sex: M\&F } \\
\text { Age: not } \\
\text { reported }\end{array}$ & $\begin{array}{l}\text { Source of } \\
\text { data: the EPA } \\
\text { Air Quality } \\
\text { System (AQS), } \\
\text { Exposure } \\
\text { assessment: } \\
\text { Satellite } \\
\text { remote } \\
\text { sensing, land } \\
\text { use, and } \\
\text { chemical } \\
\text { transport } \\
\text { models } \\
\text { Type: Annual } \\
\text { average of } \\
\text { PM }_{2.5}\end{array}$ & $\begin{array}{l}\text { Number of } \\
\text { cases: } 570,668 \\
\text { Diagnose: } \\
\text { physician- } \\
\text { diagnosed } \\
\text { cases of MI } \\
\text { based on } \\
\text { recorded } \\
\text { obtained from } \\
\text { the Center for } \\
\text { Medicare and } \\
\text { Medicaid } \\
\text { Services } \\
\text { denominator } \\
\text { file according } \\
\text { to ICD-9: } 410\end{array}$ & $\begin{array}{l}\text { Index: HR( }(95 \% \mathrm{Cl}) \text { per } \\
1 \mu \mathrm{m} / \mathrm{m}^{3} \text { increment } \\
\text { of PM } \\
\text { Adjustment: sex, } \\
\text { race, state, Medicaid } \\
\text { eligibility, ozone, year, } \\
\text { median house value, } \\
\text { percent owner } \\
\text { occupied housing, } \\
\text { percent living below } \\
\text { poverty, population } \\
\text { density, and median } \\
\text { household income. }\end{array}$ & Gooc \\
\hline
\end{tabular}

\subsection{Risk of bias in individual studies}

We assessed the selected studies in terms of risk of bias. The Newcastle-Ottawa Scale (NOS) developed for investigating the quality of cohort studies was used ${ }^{45}$. The NOS is largely used due to recommendations from the Cochrane Collaboration ${ }^{46}$. This tool has three broad categories, including selection (four criteria), comparability of study groups (two criteria), and assessment of the outcome (three criteria). The scale ranged between 0 (the lowest study quality) and 
9 (the highest study quality) points. The final quality score according to the NOS was provided in the Table 1 for each included study.

\subsection{Summary measures}

We included only prospective cohort studies. All studies were reported HR and 95\% Cl for developing of MI per specific unit increase in PM levels. For estimating the total effect of exposure to PM on the risk of Ml, because some studies reported $\mathrm{HR}(95 \% \mathrm{Cl})$ for $\mathrm{PM}_{10}, \mathrm{PM}_{2.5}$, or/and $\mathrm{PM}_{2.5-10}{ }^{30,35,39}$, we only included the estimated risk per $\mathrm{PM}_{10}$ due to overlapping. Moreover, we found two studies reported HR of MI for different PM diameters on the same population ${ }^{40-41}$ For total effect analysis, we only considered one of them reported HR of MI for $\mathrm{PM}_{10}{ }^{40}$ but for subgroup analyse according to PM diameters we included both studies ${ }^{40-41}$. Similarly, there were two studies on the same population and PM diameter ${ }^{27-28}$. We first used fixed effects meta-analysis model, then the estimated pooled $\mathrm{HR}(95 \% \mathrm{Cl})$ was considered. In the present study, we assumed a linear relation between PM air pollution and MI. If applicable, we standardized the risk of MI per $1 \mu \mathrm{g} / \mathrm{m}^{3}$ increment of PM air pollution by using the following formula:

Standardized HR $(95 \% \mathrm{CI})$ of $\mathrm{MI}=\mathrm{e}^{\left(\operatorname{Ln}\left(\text { Original Estimated HR[95\% CI])* } \frac{1}{\text { Original increment }}\right)\right.}$

\subsection{Synthesis of results}

The Random-effects model through a generic inverse-variance method was used to calculate the pooled $\mathrm{HR}(95 \% \mathrm{Cl})$ for incidence of $\mathrm{MI}^{47}$. Heterogeneity presented with calculated $\mathrm{I}^{2}$ index and $\mathrm{I}^{2}$ values of $0 \%, 25 \%, 50 \%$,and $75 \%$ represent no, low, moderate, and high heterogeneity, respectively ${ }^{48-49}$. We used the Jackknife approach to investigate the impact of each study on the pooled effect size and the heterogeneity across studies ${ }^{52}$. Besides, Subgroup analyses according to gender (female or both male and female subjects, study population (subjects without MI history and those with $\mathrm{MI} / \mathrm{CHD}$ history), and diameter of $\mathrm{PM}\left(\mathrm{PM}_{2.5}, \mathrm{PM}_{2.5-10}\right.$, and $\left.\mathrm{PM}_{10}\right)$ were performed. P-value of less than 0.05 was chosen to test null hypotheses in all analysis. Stata software version 14 was used to data analysis.

\subsection{Risk of bias across studies}

The Egger's and bagger's tests employed to investigate publication bias ${ }^{50-51}$. The p-value less than 0.05 chosen to test a significant publication bias across studies. In addition, Visual inspection of funnel plot was presented.

\section{Results}

\subsection{Study selection}

Flow diagram of systematic review process was presented in the Fig. 1. Totally, search in selected databases was derived to identify 3190 literature. After removing duplicate records, we screened 1795 records by title and abstract. Then, we retrieved the full text of 430 potentially relevant articles. After excluding 413 papers by reasons, eventually, the number of 17 prospective cohort studies entered in this systematic review and meta-analysis ${ }^{26-42}$.

\subsection{Study characteristics}

As provided in Supplemental Table 1, we included the number of 17 prospective cohort studies with more than 18 million participants and 800,000 cases of Ml. The large number of the included studies (10 out of 17) was performed in North America region (the eight studies in the United States of America and the two studies in Canada). Besides, there were 
four studies came from Europa region (two studies from the United Kingdom, another study from Netherlands, and the other study from Italy), two studies from East Asia (one study from Korea and another study from Japan), and one study from Middle East (Israel). All studies carried out on adult subjects (the youngest age was $\geq 18$ years). Most of the studies included both female and male subjects but in the four studies participants had female gender. In the 14 studies only subjects without CVDs included but in the three studies participants had previous history of MI or CVDs. Most studies used the different models to determine the rate of exposure to PM for the subjects (12 studies). By considering the aerodynamic diameter of particulates, we observed in the nine studies only $\mathrm{PM}<2.5 \mu \mathrm{m}$ investigated and in the three studies only $\mathrm{PM} \leq 10 \mu \mathrm{m}$ considered. Furthermore, in the other three studies assessed both $\mathrm{PM}<2.5$ and $\mathrm{PM} \leq 10 \mu \mathrm{m}$. Moreover, one study investigated $\mathrm{PM}<2.5$ and PM 2.5-10 (Coarse) and in the other in addition to PM $<2.5$ and PM 2.5$10 \mu \mathrm{m}$ included $\mathrm{PM} \leq 10 \mu \mathrm{m}$. the majority of included studies diagnosed MI based on medical records registers by ICD-9 codes 140 (three studies) and/or ICD-10 codes I21(three studies) or I21-23(four studies). All studies provided the adjusted hazard ratio (HR) and $95 \%$ confidence interval $(\mathrm{Cl})$ of per specific unit increment of PM.

\subsection{Risk of bias within studies}

The results of risk of according to the NOS tool indicates all included studies have good quality (all studies met $3 / 4$ items of Selection part, 1/2 items of Comparability par, and 2/3 items of Outcome part).

\subsection{Results of individual studies}

The results of studies were differed according to type of PM. OF the 13 studies investigated the risk of MI per specific unit increment of $\mathrm{PM} \leq 2.5 \mu \mathrm{m}$, seven studies reported a higher risk of $\mathrm{Ml}$ that in the most of them (five studies) the risk was significant. Besides, in the other six studies did not show a higher risk of MI that even in a study the risk of MI decreased per specific unit increment of $\mathrm{PM} \leq 2.5 \mu \mathrm{m}$. for particulates with aerodynamic range of 2.5 to $10 \mu \mathrm{m}$, of $3 \mathrm{six}$ studies investigated association, the number of two studies found a significantly higher risk of $\mathrm{Ml}$ and another study did not observe a higher risk of MI per increasing in the PM. In related to PM $\leq 10 \mu \mathrm{m}$, we found 6 studies examined the relationship. Of three studies reported a higher risk of $\mathrm{Ml}$, the risk of $\mathrm{MI}$ was significant in two studies. Moreover, in the other three studies did not find a higher risk of MI per increment of PM.

\subsection{Synthesis of results}

As showed in Fig. 2, the pooled HR $(95 \% \mathrm{Cl})$ of $\mathrm{Ml}$ per $1 \mu \mathrm{g} / \mathrm{m}^{3}$ increment of $\mathrm{PM}_{10}$ was estimated at 1.02(1.01, 1.03). Furthermore, we observed a significantly high heterogeneity across studies $\left(I^{2}=96.8 \%[p\right.$-value $\left.<0.001]\right)$. By considering the study population (subjects without MI history and those with $\mathrm{MI} / \mathrm{CHD}$ history), As showed in Fig. 3, the pooled HR $(95 \% \mathrm{Cl})$ per $1 \mu \mathrm{g} / \mathrm{m} 3$ increase in PM for studies considered subjects without MI history and those with MI/CHD history were calculated 1.02(1.00, 1.03) and 1.05(1.01, 1.08). In addition, $I^{2}$ (P-value) were $94.7(<0.001)$ and $16.1 \%(0.303)$, respectively. We also performed subgroup analysis according to gender, including female and both genders. As plotted in Supplemental Fig. 2, there was no significant higher risk of Ml in female subgroup, but we found a significantly higher risk of $\mathrm{MI}$ in the other subgroup. Apart from, we used the Jackknife approach to investigate the impact of each study on the pooled effect size and the heterogeneity across studies (see Fig. 4). After excluding Kim et al study as presented in Fig. 5, we again found a significantly higher risk of MI per $1 \mu \mathrm{g} / \mathrm{m}^{3}$ increase in $\mathrm{PM}_{10}(\mathrm{HR}=1.01,95 \% \mathrm{CI}[1.00,1.02])$, but with a slightly lower heterogeneity $\left(I^{2}=91.7 \%[P-v a l u e<0.001]\right)$. Furthermore, subgroup analyses according to the aerodynamic diameter of PM was carried out. Accordingly, we classified studies into three subgroups including $\mathrm{PM}_{2.5}$, $\mathrm{PM}_{2.5-10}$, and $\mathrm{PM}_{10}$. As presented in Fig. 6, the pooled $\mathrm{HR}(95 \% \mathrm{Cl})$ per $1 \mu \mathrm{g} / \mathrm{m}^{3}$ increase in $\mathrm{PM}_{2.5}$ was estimated 1.01 $(1.00,1.02)$ with $I^{2}=91.1 \%(P$-value $<0.001)$. It remains significant after excluding subjects with MI/CHD history (See Supplemental Fig. 3). Per $1 \mu \mathrm{g} / \mathrm{m}^{3}$ increase, the pooled HR $(95 \% \mathrm{Cl})$ and I2 (P-value) were calculated $1.40(0.87,1.94)$ and $99.3(p<0.001)$ for $\mathrm{PM}_{2.5-10}$ and $1.01(0.99,1.02)$ and $67.3(p=0.009)$. It should be noted we observed the same results for $\mathrm{PM}_{10}$ after subgroup analysis based on type of population (Supplemental Fig. 4). 


\subsection{Risk of bias across studies}

We did not show a significant publication bias across studies according to both Begg's Test (P-value $=0.102)$ Egger's test (P-value $=0.729)$. The funnel plot also was provided in supplemental Fig. 1.

\section{Discussion}

According to the best of our knowledge, this systematic review and meta-analysis study was the first attempt to investigate the effect of long-term exposure to PM air pollution on the incidence of MI. Overall, The number of 17 prospective cohort studies with more than 18 million participants and 800,000 cases of MI was included. Obtained results indicated per $1 \mu \mathrm{g} / \mathrm{m}^{3}$ increment of $\mathrm{PM}_{10}$, the risk of $\mathrm{MI}$ raised significantly by $2 \%$ averagely. This finding was in line with some studies found a significantly greater risk of CVDs results from long-term exposure to PM $8,12,53$. Besides, in comparison to previous meta-analysis investigated the short-term effect of exposure to $\mathrm{PM}_{10}$ on the risk of $\mathrm{MI}$, we observed stronger association between $\mathrm{PM}$ and $\mathrm{MI}^{22-25}$. Furthermore, subgroup analysis based on types of study population reveals subjects with $\mathrm{Ml} / \mathrm{CHD}$ history in comparison to who without MI history had a significantly $3 \%$ greater risk of MI per $1 \mu \mathrm{g} / \mathrm{m}^{3}$ increase in the levels of PM averagely. Apart from this, by considering aerodynamic diameter of $\mathrm{PM}$, We found only a significant stronger risk of MI per $1 \mu \mathrm{g} / \mathrm{m}^{3}$ increase in PM with aerodynamic diameter less than 2.5 $\mu \mathrm{m}$, but not for $\mathrm{PM}_{2.5-10}$ and $\mathrm{PM}_{10}$. This finding was in line of several previous studies reported a significantly association between $\mathrm{PM}_{2.5}$ and CVD influence and mortality ${ }^{12,13,54}$. This finding also confirms the greater effect of the smaller size fractions of PM on the cardiovascular system ${ }^{2,55}$. In sum, this study indicates a significant association between long-term exposure to $\mathrm{PM} \leq 10 \mu \mathrm{g} / \mathrm{m}^{3}$ and the developing of Ml. Although biological mechanisms underlining the association not fully understood, the possible biological pathways were discussed with details in several invaluable studies ${ }^{2,56-59}$. Briefly, PM air pollution can result in impairments in the cardiovascular system, including heart, vascular, and blood through several pathways, including lung inflammation, neuroendocrine activation, particle translocation, and undefined bloodborne mediator. It is supposed that in long-term exposure to PM, these impairments can raise the developing of cardiovascular morbidity and mortality ${ }^{2}$.

This study accompanied with several advantages. This study was the first attempt to quantify the long-term effect of exposure to PM on the incidence of MI. We included the prospective cohort studies with appropriate sample size (more than 18 million participants), cases of $\mathrm{Ml}$ (more than 800,000 cases). However, there are some shortcomings in the both study and outcome levels that should be taken into account when interpreting the results of this review. First of all, for measuring exposure to PM, the included studies applied various methods having different precision. Moreover, most studies did not determine exposure to PM in other situations such as indoor and occupational sources. Apart from, PM health effects can be vary depending on its nature. Studies did not account this and only considered the diameter of PM. The studies provided adjusted HR $(95 \% \mathrm{Cl})$ of $\mathrm{MI}$ per different increases in PM levels. Thus, we assumed a linear relation between PM and $\mathrm{Ml}$ and standardized the risk of MI per $1 \mu \mathrm{g} / \mathrm{m} 3$ increase in PM levels. This also can be associated with error in measuring the effects size of association between. Second, the definition of MI across studies is problematic. Some studies used ICD codes but the others did not. Among studies provided ICD codes, some studies defined MI as ICD9: 410 or ICD-10:I21 (primary MI) but the others considered both primary and secondary types of MI and applied ICD-10: 121-23. Last but not the least, we found a high heterogeneity across studies. What is more, in the large number of included studies, MI incidence data obtained from registry information. This can potentially increase the risk of nondifferential bias because of coding errors. Third, confounding effect is another important limitation across studies. Recent meta-analysis study has indicated that exposure to environmental noise can be associated with higher risk of MI

60. The most selected studies did not adjust this effect. None of the included studies did not adjust exposure to occupational risk factor such as noise, shift work and life style risk factors such as sleep status, diets patterns, and

Page $15 / 24$ 
physical activity that according to previous studies can be associated with the higher odds of MI. Forth, combining HR $(95 \% \mathrm{Cl})$ adjusted for different covariates may have reduced the consistency across studies and reduced the precision of our summary estimates. Finally, although subgroup analyses based on type of population and sensitivity analyses according to Jackknife approach were performed, we found a high heterogeneity, especially in studies conducted among subjects without history of MI. More studies are needed to explore the sources of heterogeneity.

\section{Conclusion}

This study was the first attempt, according to the best of our knowledge, to investigate the effect of long-term exposure to PM air pollution on the risk of MI. Obtained results indicated a significantly higher risk of MI per $1 \mu \mathrm{g} / \mathrm{m} 3$ increase in the level of $\mathrm{PM}_{10}$. Moreover, Subgroup analysis based on types of study population reveals subjects with $\mathrm{Ml} / \mathrm{CHD}$ history in comparison to who without MI history had a significantly greater risk of MI per $1 \mu \mathrm{g} / \mathrm{m} 3$ increase in the levels of $P M_{10}$. Considering aerodynamic diameter of PM shows only a significant stronger risk of MI per $1 \mu \mathrm{gg} / \mathrm{m} 3$ increase in $P M_{2.5}$. More studies are warranted to overcome the above-mentioned limitations and confirm our findings.

\section{Declarations}

\section{Acknowledgments: None.}

Author contributions: All authors contributed to the study conception and design. Design of the work raised by [M.Kh]. Material preparation, data collection and analysis were performed by [R.S], [F.O] and [F.R]. The first draft of the manuscript was written by [M.Kh] and all authors commented on previous versions of the manuscript. All authors read and approved the final manuscript.

Funding: This study was approved by Research Deputy and Technology of Kermanshah University of Medical Sciences.

Declaration of Conflicting Interest: The authors declare they have no actual or potential conflicts of interest with respect to the research, authorship, and/or publication of this article.

\section{References}

1. World_Health_Organization Air Pollution. doi:https://www.who.int/airpollution/ en/ (29 August 2019, date last accessed).

2. Miller, M. R. \& Newby, D. E. Air pollution and cardiovascular disease: car sick. Cardiovasc Res. 116, 279-294 (2020).

3. GBD 2016 Risk Factors Collaborators. Global, regional, and national comparative risk assessment of 84 behavioural, environmental and occupational, and metabolic risks or clusters of risks, 1990-2016: a systematic analysis for the Global Burden of Disease Study 2016. Lancet. 390, 1345-1422 (2017).

4. Li, M. H. et al. Short-term Exposure to Ambient Fine Particulate Matter Increases Hospitalizations and Mortality in COPD: A Systematic Review and Meta-analysis. Chest. 149, 447-458 (2016).

5. Orellano, P., Quaranta, N., Reynoso, J., Balbi, B. \& Vasquez, J. Effect of outdoor air pollution on asthma exacerbations in children and adults: Systematic review and multilevel meta-analysis. PLoS One. 20, 120174050 (2017).

6. Huang, F., Pan, B., Wu, J., Chen, E. \& Chen, L. Relationship between exposure to PM2.5 and lung cancer incidence and mortality: A meta-analysis. Oncotarget. 8, 43322-43331 (2017).

7. Shah, A. S. et al. Global association of air pollution and heart failure: a systematic review and meta-analysis. Lancet. 382, 1039-1048 (2013). 
8. Cai, Y. et al. Associations of Short-Term and Long-Term Exposure to Ambient Air Pollutants With Hypertension: A Systematic Review and Meta-Analysis. Hypertension. 68 (1), 62-70 (2016 Jul).

9. Akintoye, E. et al. Association between fine particulate matter exposure and subclinical atherosclerosis: A metaanalysis. Eur J Prev Cardiol. 23, 602-612 (2016).

10. Song, X. et al. Short-Term Exposure to Air Pollution and Cardiac Arrhythmia: A Meta-Analysis and Systematic Review. Int J Environ Res Public Health. 13, 642 (2016).

11. Liu, X. et al. Association of Exposure to particular matter and Carotid Intima-Media Thickness: A Systematic Review and Meta-Analysis. Int J Environ Res Public Health. 12, 12924-12940 (2015).

12. Yuan, S. et al. Long-term exposure to PM2.5 and stroke: A systematic review and meta-analysis of cohort studies. Environ Res. 177, 108587 (2019).

13. Shin, H. H., Fann, N., Burnett, R. T., Cohen, A. \& Hubbell, B. J. Outdoor fine particles and nonfatal strokes: systematic review and meta-analysis. Epidemiology. 25, 835-842 (2014).

14. Yang, M. et al. Effects of long-term exposure to air pollution on the incidence of type 2 diabetes mellitus: a metaanalysis of cohort studies. Environ Sci Pollut Res Int. 27, 798-811 (2020).

15. Liu, F. et al. Associations between long-term exposure to ambient air pollution and risk of type 2 diabetes mellitus: A systematic review and meta-analysis. Environ Pollut. 252, 1235-1245 (2019).

16. Fu, P., Guo, X., Cheung, F. M. H. \& Yung, K. K. L. The association between PM2.5 exposure and neurological disorders: A systematic review and meta-analysis. Sci Total Environ. 655, 1240-1248 (2019).

17. Zeng, Y., Lin, R., Liu, L., Liu, Y. \& Li, Y. Ambient air pollution exposure and risk of depression: A systematic review and meta-analysis of observational studies. Psychiatry Res. 276, 69-78 (2019).

18. Wu, M. Y., Lo, W. C., Chao, C. T., Wu, M. S. \& Chiang, C. K. Association between air pollutants and development of chronic kidney disease: A systematic review and meta-analysis. Sci Total Environ. 706, 135522 (2020).

19. Zhang, Z., Yan, W., Chen, Q., Zhou, N. \& Xu, Y. The relationship between exposure to particulate matter and breast cancer incidence and mortality: A meta-analysis. Medicine. 98, e18349 (2019).

20. Newell, K., Kartsonaki, C., Lam, K. B. H. \& Kurmi, O. P. Cardiorespiratory health effects of particulate ambient air pollution exposure in low-income and middle-income countries: a systematic review and meta-analysis. Lancet Planet Health. 1, e368-e380 (2017).

21. Burnett, R. et al. Global estimates of mortality associated with long-term exposure to outdoor fine particulate matter 1159592-9597(Proc Natl Acad Sci U S A, 2018).

22. Farhadi, Z., Abulghasem Gorgi, H., Shabaninejad, H., Aghajani Delavar, M. \& Torani, S. Association between PM2.5 and risk of hospitalization for myocardial infarction: a systematic review and a meta-analysis. BMC Public Health. 20, 314 (2020).

23. Cai, X., Li, Z., Scott, E. M., Li, X. \& Tang, M. Short-term effects of atmospheric particulate matter on myocardial infarction: a cumulative meta-analysis. Environ Sci Pollut Res Int. 23, 6139-6148 (2016).

24. Luo, C. et al. Short-term exposure to particulate air pollution and risk of myocardial infarction: a systematic review and meta-analysis. Environ Sci Pollut Res Int. 22, 14651-14662 (2015).

25. Mustafic, H. et al. Main air pollutants and myocardial infarction: a systematic review and meta-analysis. JAMA. 307, 713-721 (2012).

26. Danesh Yazdi, M., Wang, Y., Di, Q., Zanobetti, A. \& Schwartz, J. Long-term exposure to PM2.5 and ozone and hospital admissions of Medicare participants in the Southeast USA. Environ Int. 130, 104879 (2019).

27. Bai, L. et al. Associations of Long-Term Exposure to Ultrafine Particles and Nitrogen Dioxide With Increased Incidence of Congestive Heart Failure and Acute Myocardial Infarction. Am J Epidemiol. 188, 151-159 (2019).

Page $17 / 24$ 
28. Bai, L. et al. Exposure to ambient air pollution and the incidence of congestive heart failure and acute myocardial infarction: A population-based study of 5.1 million Canadian adults living in Ontario. Environ Int. 132, 105004 (2019).

29. Gandini, M. et al. Long term effect of air pollution on incident hospital admissions: Results from the Italian Longitudinal Study within LIFE MED HISS project. Environ Int. 121, 1087-1097 (2018).

30. Kim, H. et al. Cardiovascular Effects of Long-Term Exposure to Air Pollution: A Population-Based Study With 900845 Person-Years of Follow-up. J Am Heart Assoc. 6, e007170 (2017).

31. Hartiala, J. et al. Ambient Air Pollution Is Associated With the Severity of Coronary Atherosclerosis and Incident Myocardial Infarction in Patients Undergoing Elective Cardiac Evaluation. J Am Heart Assoc. 5, e003947 (2016).

32. Nishiwaki, Y. et al. Long-term exposure to particulate matter in relation to mortality and incidence of cardiovascular disease: the JPHC Study. J Atheroscler Thromb. 20, 296-309 (2013).

33. Zanobetti, A. \& Schwartz, J. Particulate air pollution, progression, and survival after myocardial infarction. Environ Health Perspect. 115, 769-775 (2007).

34. Loop, M. S. et al. Fine particulate matter and incident coronary heart disease in the REGARDS cohort. Am Heart J. 197, 94-102 (2018).

35. Downward, G. S. et al. Long-Term Exposure to Ultrafine Particles and Incidence of Cardiovascular and Cerebrovascular Disease in a Prospective Study of a Dutch Cohort. Environ Health Perspect. 126 (12), 127007 (2018 Dec).

36. Carey, I. M. et al. Traffic pollution and the incidence of cardiorespiratory outcomes in an adult cohort in London. Occup Environ Med. 73, 849-856 (2016).

37. Koton, S. et al. Cumulative exposure to particulate matter air pollution and long-term post-myocardial infarction outcomes. Prev Med. 57, 339-344 (2013).

38. Atkinson, R. W. et al. Long-term exposure to outdoor air pollution and incidence of cardiovascular diseases. Epidemiology. 24, 44-53 (2013).

39. Lipsett, M. J. et al. Long-term exposure to air pollution and cardiorespiratory disease in the California teachers study cohort. Am J Respir Crit Care Med. 184, 828-835 (2011).

40. Puett, R. C. et al. Chronic fine and coarse particulate exposure, mortality, and coronary heart disease in the Nurses' Health Study. Environ Health Perspect. 117, 1697-1701 (2009).

41. Puett, R. C. et al. Chronic particulate exposure, mortality, and coronary heart disease in the nurses' health study. Am J Epidemiol. 15, 1681161-1681168 (2008).

42. Miller, K. A. et al. Long-term exposure to air pollution and incidence of cardiovascular events in women. N Engl J Med. 1, 356447-356458 (2007).

43. Moher, D. et al. Preferred reporting items for systematic review and meta-analysis protocols (PRISMA-P) 2015 statement. Syst Rev. 4, 1 (2015).

44. iberati, A. et al. The PRISMA statement for reporting systematic reviews and meta-analyses of studies that evaluate health care interventions: explanation and elaboration. PLoS Med. 6, e1000100 (2009).

45. Wells, G. A., O'Connell, B. S. D., Peterson, J., Welch, V. \& Losos, M. P Tugwell. The Newcastle-Ottawa Scale (NOS) for assessing the quality of nonrandomised studies in meta-analyses.

46. Higgins, J. \& Wells, G. Cochrane handbook for systematic reviews of interventions 2011.

47. DerSimonian, R. \& Laird, N. Meta-analysis in clinical trials revisited. Contemp Clin Trials. 45, 139-145 (2015).

48. Higgins, J. P. \& Thompson, S. G. Quantifying heterogeneity in a meta-analysis. Stat Med. 21, 1539-1558 (2002). 
49. Higgins, J. P., Thompson, S. G., Deeks, J. J. \& Altman, D. G. Measuring inconsistency in meta-analyses. BMJ. 327, 557-560 (2003).

50. Begg, C. B. \& Mazumdar, M. Operating characteristics of a rank correlation test for publication bias. Biometrics. 50, 1088-1101 (1994).

51. Egger, M., Davey Smith, G., Schneider, M. \& Minder, C. Bias in meta-analysis detected by a simple, graphical test. BMJ. 315, 629-634 (1997).

52. Miller, R. G. The jackknife-a review.Biometrika174; 61,1-15.

53. Cesaroni, G. et al. A. Long term exposure to ambient air pollution and incidence of acute coronary events: prospective cohort study and meta-analysis in 11 European cohorts from the ESCAPE Project. Version 2. BMJ.;348:f7412.

54. Liu, Z. et al. Does utilizing WHO's interim targets further reduce the risk - meta-analysis on ambient particulate matter pollution and mortality of cardiovascular diseases? Environ Pollut. 242, 1299-1307 (2018).

55. Ohlwein, S., Kappeler, R., Kutlar Joss, M., Künzli, N. \& Hoffmann, B. Health effects of ultrafine particles: a systematic literature review update of epidemiological evidence. Int J Public Health. 64, 547-559 (2019).

56. Rajagopalan, S., Al-Kindi, S. G. \& Brook, R. D. Air Pollution and Cardiovascular Disease: JACC State-of-the-Art Review. J Am Coll Cardiol. 72, 2054-2070 (2018).

57. Fiordelisi, A. et al. The mechanisms of air pollution and particulate matter in cardiovascular diseases. Heart Fail Rev. 22, 337-347 (2017).

58. Franchini, M., Guida, A., Tufano, A. \& Coppola, A. Air pollution, vascular disease and thrombosis: linking clinical data and pathogenic mechanisms. J Thromb Haemost. 10, 2438-2451 (2012).

59. Brook, R. D. et al. Council on the Kidney in Cardiovascular Disease, and Council on Nutrition, Physical Activity and Metabolism. Particulate matter air pollution and cardiovascular disease: An update to the scientific statement from the American Heart Association. Circulation. 121, 2331-2378 (2010).

60. Khosravipour, M. \& Khanlari, P. The association between road traffic noise and myocardial infarction: A systematic review and meta-analysis. Sci Total Environ. 731, 139226 (2020).

\section{Figures}


Papers identified through database searching $(n=3190)$

- Scopus $(\mathrm{n}=1043)$

- Web of science $(n=1325)$

- PubMed $(\mathrm{n}=822)$
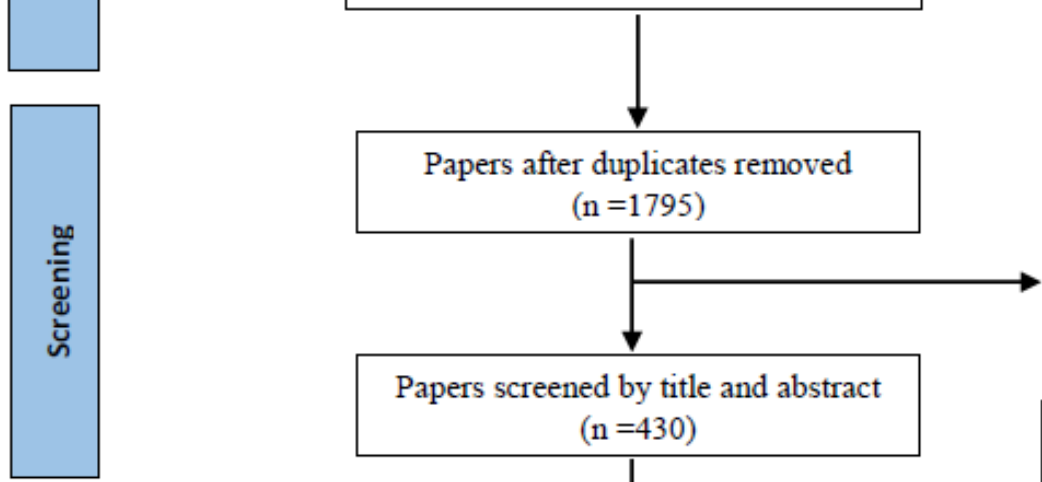

Papers excluded by title and abstract $(\mathrm{n}=1367)$

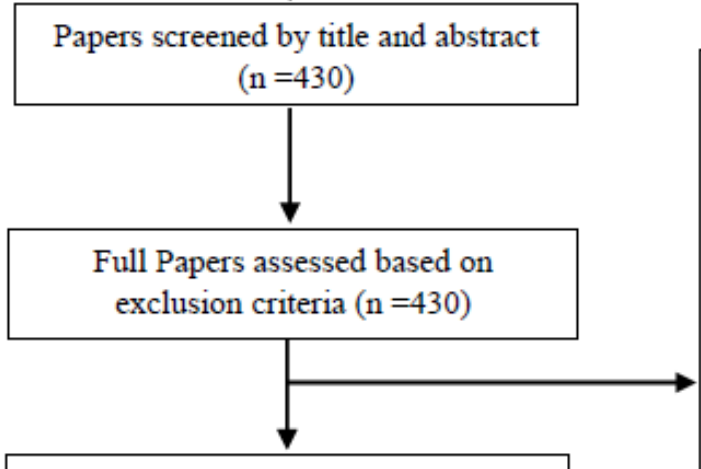

Full Papers assessed based quality appraisal $(\mathrm{n}=17)$

Papers excluded by with reasons $(n=413)$

Did not assess the relation: 131

Short-term or retrospective studies: 163

Review, conference, and editorial papers: 82

No particulate matter: 11

No myocardial infarction (MI): 9

MI mortality: 8

Mixed MI incidence and mortality: 2

Duplicates: 3

Occupational sitting: 1

Not only MI: 3

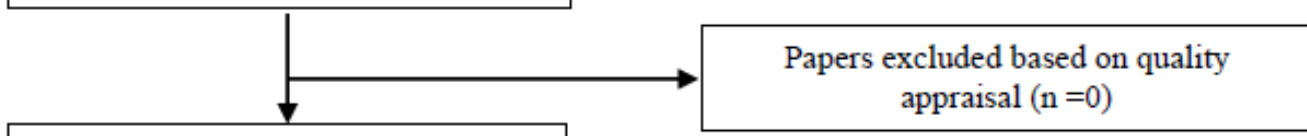

Prospective cohort Studies included in systematic review $(\mathrm{n}=17)$
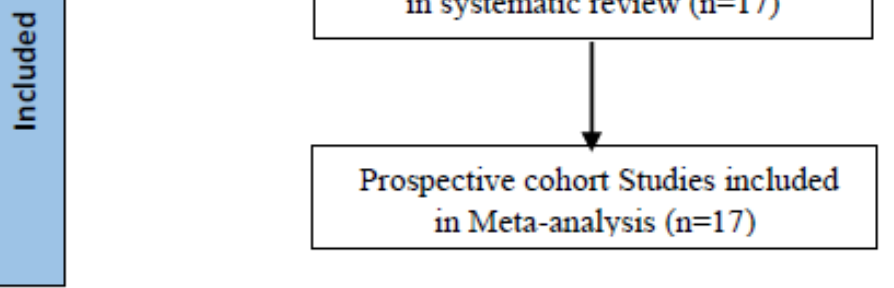

Figure 1

Flow diagram of systematic review process. 


\begin{tabular}{|c|c|c|}
\hline Study ID & $\mathrm{HR}(95 \% \mathrm{Cl})$ & Weight \% \\
\hline Miller (2007) & $1.01(0.98,1.03)$ & 8.10 \\
\hline Zanobetti (2007) & $1.04(1.01,1.06)$ & 7.58 \\
\hline Puett (2008) & $0.99(0.97,1.01)$ & 8.51 \\
\hline Lipsett (2011) & $1.00(0.99,1.01)$ & 10.99 \\
\hline Atkinson (2013) & $0.99(0.98,1.00)$ & 10.22 \\
\hline Koton (2013) & $1.05(0.99,1.11)$ & 2.91 \\
\hline Nishiwaki (2013) & $1.03(1.00,1.06)$ & 6.80 \\
\hline Carey (2016) & $0.88(0.79,0.98)$ & 1.41 \\
\hline Hartiala (2016) & $1.14(1.01,1.28)$ & 0.75 \\
\hline Kim (2017) & $\rightarrow 1.90(1.79,2.00)$ & 1.21 \\
\hline Gandini (2018) & $1.01(1.01,1.02)$ & 11.40 \\
\hline Loop (2018) & $0.94(0.89,1.00)$ & 3.56 \\
\hline Bai (2019) & $1.01(1.01,1.01)$ & 11.40 \\
\hline Yazdi (2019) & $1.03(1.02,1.03)$ & 11.44 \\
\hline Downward (2018) & $1.02(0.97,1.08)$ & 3.71 \\
\hline Overall (I-squared $=96.8 \%, p=0.000)$ & $1.02(1.01,1.03)$ & 100.00 \\
\hline NOTE: Weights are from random effects analysis & & \\
\hline
\end{tabular}

\section{Figure 2}

Forest plot for the association between Particulate matter (PM ) air pollution with aerodynamic $\leq 10 \mu \mathrm{m}$ and the risk of myocardial infarction (MI). HR, hazard ratio; $\mathrm{Cl}$, confidence interval. 


\begin{tabular}{|c|c|c|}
\hline Study ID & $\mathrm{HR}(95 \% \mathrm{Cl})$ & Weight $\%$ \\
\hline \multicolumn{3}{|l|}{ Subjects without CVDs history } \\
\hline Miller (2007) & $1.01(0.98,1.03)$ & 9.12 \\
\hline Puett (2008) & $0.99(0.97,1.01)$ & 9.59 \\
\hline Lipsett (2011) & $1.00(0.99,1.01)$ & 12.38 \\
\hline Atkinson (2013) & $0.99(0.98,1.00)$ & 11.51 \\
\hline Nishiwaki (2013) & $1.03(1.00,1.06)$ & 7.66 \\
\hline Carey $(2016)$ & $0.88(0.79,0.98)$ & 1.59 \\
\hline Kim (2017) & $\Rightarrow 1.90(1.79,2.00)$ & 1.37 \\
\hline Gandini (2018) & $1.01(1.01,1.02)$ & 12.84 \\
\hline Loop (2018) & $0.94(0.89,1.00)$ & 4.02 \\
\hline Bai (2019) & $1.01(1.01,1.01)$ & 12.84 \\
\hline Yazdi (2019) & $1.03(1.02,1.03)$ & 12.89 \\
\hline Downward (2018) & $1.02(0.97,1.08)$ & 4.18 \\
\hline Subtotal $(I-$ squared $=97.4 \%, p=0.000)$ & $1.02(1.00,1.03)$ & 100.00 \\
\hline \multicolumn{3}{|l|}{ Subjects with CVDs history } \\
\hline Zanobetti (2007) & $1.04(1.01,1.06)$ & 72.64 \\
\hline Koton (2013) & $1.05(0.99,1.11)$ & 22.18 \\
\hline Hartiala (2016) & $1.14(1.01,1.28)$ & 5.18 \\
\hline Subtotal $(I-$ squared $=16.1 \%, p=0.303)$ & $1.05(1.01,1.08)$ & 100.00 \\
\hline NOTE: Weights are from random effects & & \\
\hline
\end{tabular}

\section{Figure 3}

Forest plot for the association between Particulate matter (PM ) air pollution with aerodynamic $\leq 10 \mu \mathrm{m}$ and the risk of myocardial infarction (MI) according to the study population (with or without CVDs). CVDs, cardiovascular diseases; HR, hazard ratio; $\mathrm{Cl}$, confidence interval. 
Meta-analysis random-effects estimates (exponential form)

Study ommited

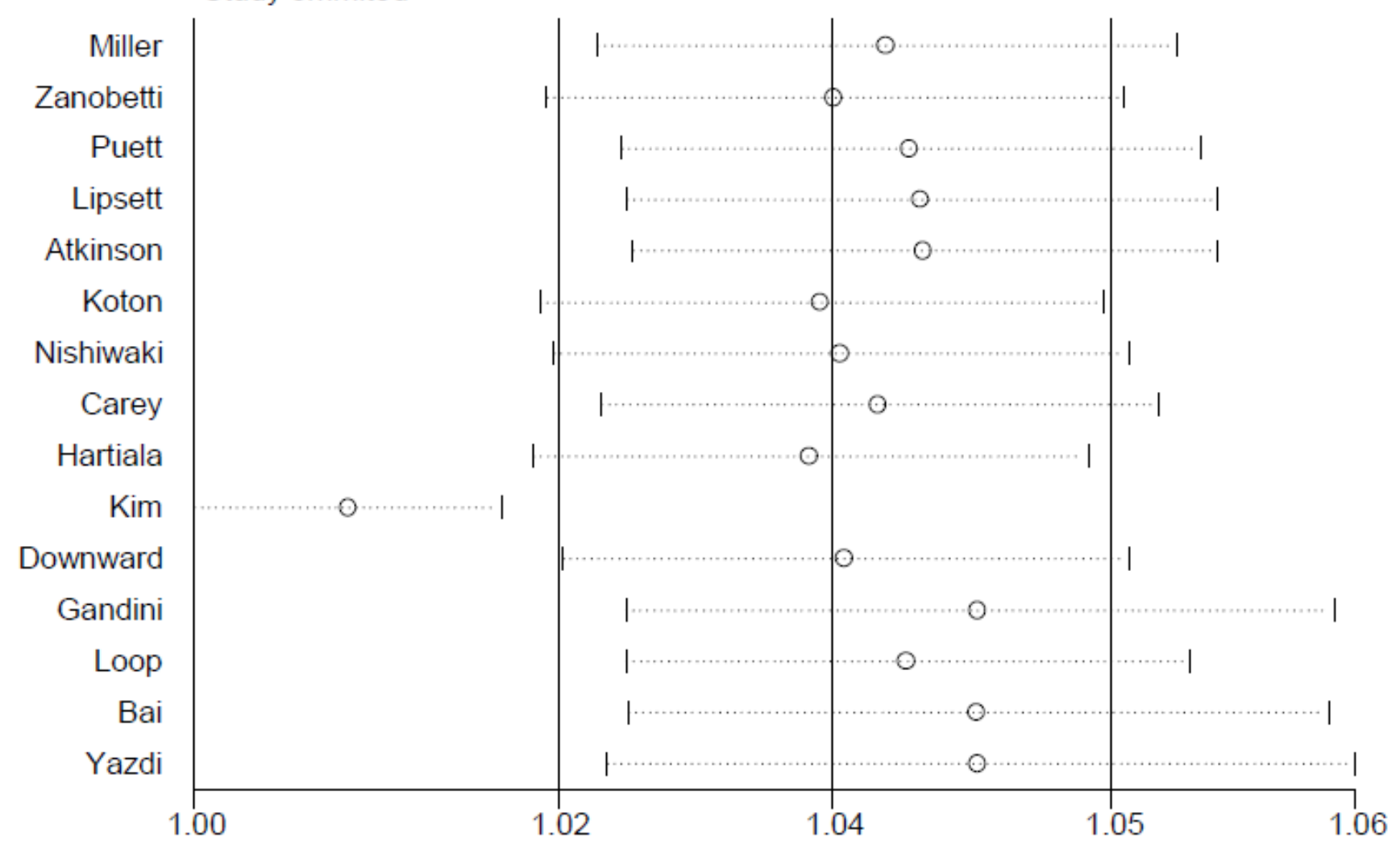

Figure 4

Sensitivity analysis using the jackknife approach.

\begin{tabular}{|c|c|c|}
\hline Study ID & $\mathrm{HR}(95 \% \mathrm{CI})$ & Weight \% \\
\hline Miller (2007) & $1.01(0.98,1.03)$ & 6.73 \\
\hline Zanobetti (2007) & $1.04(1.01,1.06)$ & 5.98 \\
\hline Puett (2008) & $0.99(0.97,1.01)$ & 7.41 \\
\hline Lipsett (2011) & $1.00(0.99,1.01)$ & 13.21 \\
\hline Atkinson (2013) & $0.99(0.98,1.00)$ & 10.98 \\
\hline Koton (2013) & $1.05(0.99,1.11)$ & 1.56 \\
\hline Nishiwaki (2013) & $1.03(1.00,1.06)$ & 4.97 \\
\hline Carey (2016) & $0.88(0.79,0.98)$ & 0.69 \\
\hline Hartiala (2016) & $1.14(1.01,1.28)$ & 0.35 \\
\hline Downward (2018) & $1.02(0.97,1.08)$ & 2.10 \\
\hline Gandini (2018) & $1.01(1.01,1.02)$ & 14.62 \\
\hline Loop (2018) & $0.94(0.89,1.00)$ & 2.00 \\
\hline Bai (2019) & $1.01(1.01,1.01)$ & 14.62 \\
\hline Yazdi (2019) & $1.03(1.02,1.03)$ & 14.79 \\
\hline Overall (l-squared $=91.7 \%, p=0.000)$ & $1.01(1.00,1.02)$ & 100.00 \\
\hline NOTE: Weights are from random effects analysis & & \\
\hline
\end{tabular}


Figure 5

Forest plot for the association between Particulate matter (PM ) air pollution with aerodynamic $\leq 10 \mu \mathrm{m}$ and the risk of myocardial infarction (MI) after removing Kim study according to Jackknife approach. $\mathrm{HR}$, hazard ratio; $\mathrm{Cl}$, confidence interval.

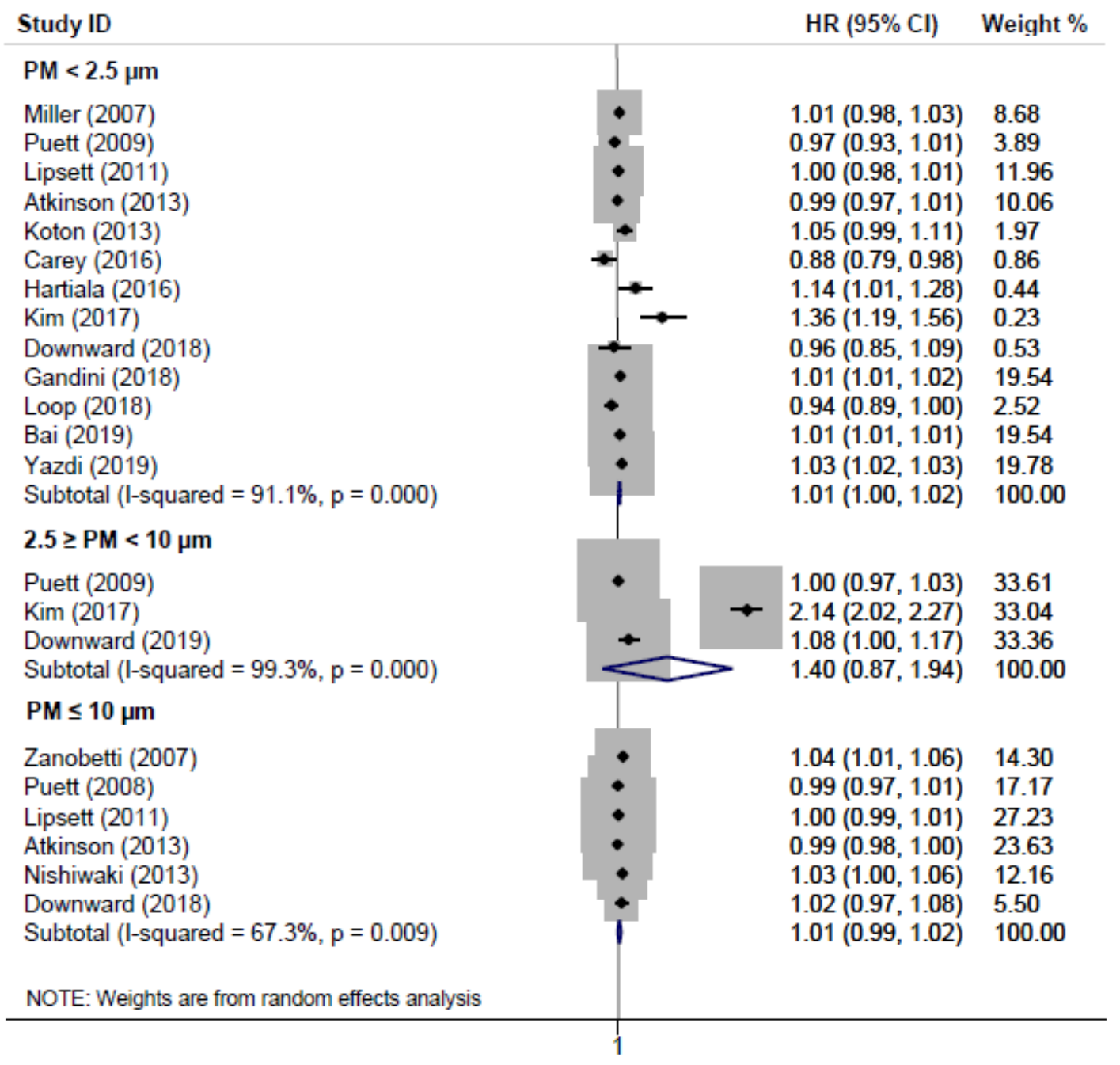

Figure 6

Forest plot for the association between Particulate matter (PM) air pollution and the risk of myocardial infarction (MI) according to PM diameters. HR, hazard ratio; $\mathrm{Cl}$, confidence interval.

\section{Supplementary Files}

This is a list of supplementary files associated with this preprint. Click to download.

- SupplementalFigure1.pdf

- SupplementalFigure2.pdf

- SupplementalFigure3.pdf

- SupplementalFigure4.pdf 